\title{
Homogeneity of differential inclusions
}

\author{
Emmanuel Bernuau, Denis Efimov, Wilfrid Perruquetti and Andrei Polyakov
}

\begin{abstract}
The notion of geometric homogeneity is extended for differential inclusions. This kind of homogeneity provides the most advanced coordinate-free framework for analysis and synthesis of nonlinear discontinuous systems. The main qualitative properties of continuous homogeneous systems are extended to the discontinuous setting: the equivalence of the global asymptotic stability and the existence of a homogeneous Lyapunov function; the link between finite-time stability and negative degree of homogeneity; the equivalence between attractivity and asymptotic stability are among the proved results.
\end{abstract}

Index Terms-Homogeneity, Differential inclusions, Lyapunov stability

\section{INTRODUCTION}

The homogeneity is an intrinsic property of an object on which the flow of a particular vector field operates as a scaling. This definition, rather simple, entails a lot of qualitative properties for a homogeneous object, and is of particular interest in view of stability purposes. The study of the stability or the asymptotic stability of a dynamical system is a central problem in the control theory. Given that the equations of a system are very often impossible to integrate explicitly, indirect methods have to be used for getting qualitative properties. Even though the results of Kurzweil [1] and Clarke [2] prove the equivalence of the asymptotic stability and the existence of a smooth Lyapunov function, finding such a Lyapunov function may be a very difficult task. Qualitative results not involving the computation of a Lyapunov function are therefore of a great interest. This is why the homogeneity theory has been developed and used in control theory: the rigid properties of homogeneous systems simplify the study of the stability and give sufficient conditions for deriving it. The literature on the homogeneity theory is vast and detailed. A lot of theoretical and practical results have been proved in the last decades, and used in different context.

However, almost all the works done for homogeneity assume continuity [3], [4], [5]. Though, throughout the last decades, interests on discontinuous systems have been increasing. The theory of differential inclusions (DI) is wellestablished [6] [7]. Among others, it appears in optimal control theory or viability theory; when dealing with variable structure systems, systems with adaptive control, power electronic systems with switching devices or mechanical systems with friction, discontinuous right-hand sides appear naturally. Finally, the sliding mode control theory makes an important use of discontinuous controller to achieve finite-time stability as well as robustness and the construction of a smooth homogeneous

E. Bernuau and W. Perruquetti are with LAGIS (UMR-CNRS 8219), Ecole Centrale de Lille, BP 48, 59651 Villeneuve D'Ascq, France

All authors are with Non-A project at INRIA Lille - Nord Europe, Parc Scientifique de la Haute Borne, 40 avenue Halley, Bt.A Park Plaza, 59650 Villeneuve d'Ascq, France
Lyapunov function is often used to prove the finite-time stability of the system, especially when dealing with higherorder sliding mode controls. Extensions of the homogeneity theory to discontinuous systems and to differential inclusions constitute therefore a natural answer to theoretical and practical problems in control theory.

Only few extensions of the homogeneity theory to DI have yet been developed in the literature. First of all, Filippov [6] defined homogeneity for DI, but only in the context of classical homogeneity. Levant [8] and Orlov [9] studied also the subject in the context of weighted homogeneity, but important properties of continuous homogeneous systems were not extended. Finally, as far as we know, except our work [10] (this paper does not contain proofs), nothing has been done about geometric homogeneity for DI.

In this paper, we shall define homogeneity for differential inclusions, connect this definition with the usual definition for vector fields, and extend all the useful properties of homogeneous ODE to homogeneous DI. We shall particularly see how the flow commutation property can be extended, demonstrate a Rosier's theorem on the existence of a Lyapunov function for globally asymptotically stable discontinuous systems and prove that the qualitative properties on homogeneous systems still apply with some slight changes. The paper is organized as follows. Section 2 will recall the notions on DI that we will use in the sequel and the usual definitions of homogeneity for vector fields. The Section 3 will be devoted to the definition and the first properties of homogeneous differential inclusions. The Section 4 will then present the main results on the qualitative properties of systems given by a homogeneous differential equation. A Conclusion will finally sum up the paper and give some directions of future works.

\section{PRELIMINARIES}

\section{A. Notations}

We endow $\mathbb{R}^{n}$ with the Lebesgue measure and denote by $\mathcal{N}$ the set of zero-measure sets. If $x \in \mathbb{R}^{n}$ and $\varepsilon>0$, the set $B(x, \varepsilon)$ denotes the Euclidian open ball centered at $x$ and of radius $\varepsilon$. The set of locally essentially bounded vector fields on $\mathbb{R}$ is denoted by $\mathcal{L}_{\text {loc }}^{\infty}\left(\mathbb{R}^{n}, \mathbb{R}^{n}\right)$. Throughout the paper, all considered vector fields belong to $\mathcal{L}_{\text {loc }}^{\infty}\left(\mathbb{R}^{n}, \mathbb{R}^{n}\right)$.

If $V: \mathbb{R}^{n} \rightarrow \mathbb{R}$ is a function (respectively $\Phi: \mathbb{R}^{n} \rightarrow \mathbb{R}$ is a diffeomorphism), we denote $d_{x} V$ the differential of the function $V$ at point $x \in \mathbb{R}^{n}$ (resp. $d_{x} \Phi$ the differential of the diffeomorphism $\Phi$ at point $x \in \mathbb{R}^{n}$ ).

\section{B. Differential inclusions}

We refer to [6] and [7] for the basic definitions and the technical material on set-valued map and differential inclusions. In 
this section, we will only recall the definitions and results that will be used hereafter, without any proof.

The Filippov's regularization procedure consists in the construction of a set-valued map $F$ starting with a vector field $f \in \mathcal{L}_{\text {loc }}^{\infty}\left(\mathbb{R}^{n}, \mathbb{R}^{n}\right)$ :

$$
\mathcal{F}[f](x)=\bigcap_{\varepsilon>0} \bigcap_{N \in \mathcal{N}} \overline{\operatorname{conv}}(f(B(x, \varepsilon) \backslash N)) .
$$

By construction, for all $x \in \mathbb{R}^{n}$, the set $\mathcal{F}[f](x)$ is closed and convex. Moreover, the set-valued map $\mathcal{F}[f]$ is upper semicontinuous.

In many applications, the differential inclusion is given by the set-valued map coming from the Filippov's procedure. We will therefore focus on set-valued map with the properties inherited by this procedure.

Definition 2.1: Let $F$ be a set-valued map. We say that $F$ verifies the Standard Assumptions (SA) if $F$ is upper semicontinuous and if for any $x \in \mathbb{R}^{n}, F(x)$ is a nonempty compact convex set. The set of set-valued maps on $\mathbb{R}^{n}$ verifying the standard assumptions is denoted by $\mathbf{F}\left(\mathbb{R}^{n}\right)$.

For DI, the standard assumptions suffice to get the existence of a solution to any Cauchy Problem. That means that any differential equation with a locally essentially bounded righthand side has a solution for any initial condition in the aforesaid sense.

In the classical setting of continuous vector fields with forward uniqueness of solutions, the flow or the semi-flow of the vector field provides a lot of qualitative informations about the system. When the forward uniqueness is lost, a flow does not exist anymore. We shall in the sequel define a generalized flow and set its properties. The proofs of these results can be found in [6] or [7].

Consider the following autonomous differential inclusion:

$$
\dot{x} \in F(x), \quad x \in \mathbb{R}^{n}, F \in \mathbf{F}\left(\mathbb{R}^{n}\right) .
$$

We say that a solution $x$ of (2) starts at $x_{0}$ if $x$ is defined on an interval containing 0 and $x(0)=x_{0}$. We will denote by $\mathcal{S}([0, T], A)$ the set of solutions of (2) defined on the interval $[0, T], T>0$, starting in $A \subset \mathbb{R}^{n}$. We also allow $T=+\infty$, and in this situation the interval $[0, T]$ has to be understood as $[0,+\infty)$. We will also denote $\mathcal{S}\left([0, T], x_{0}\right)=\mathcal{S}\left([0, T],\left\{x_{0}\right\}\right)$.

Let $T \in] 0,+\infty]$ be such that every solution of (2) starting in $A$ is defined on $[0, T]$. We denote $\Psi^{T}(A)=\{x(T): x \in$ $\mathcal{S}([0, T], A)\}$. This set is the reachable set from $A$ at time $T$, or the limit in case $T=+\infty$. Let us stress that with the assumption of uniqueness of solutions in forward time, $\Psi^{t}$ corresponds to the semiflow of $F$; this remark justifies that we call $\Psi$ the generalized flow of $F$.

Theorem 2.2: Let $K \subset \mathbb{R}^{n}$ be compact. There exists $T_{0}>0$ such that all the solutions of (2) starting in $K$ are defined on the whole interval $\left[0, T_{0}\right]$. Moreover, for all $T>0$ such that all the solutions of (2) starting in $K$ are defined on the whole interval $[0, T]$, there exists a compact set $L \subset \mathbb{R}^{n}$ such that for all $t \in[0, T], \Psi^{t}(K) \subset L$ and the set $\Psi^{t}(K)$ is compact.

Corollary 2.3: Let $T>0$ be such that all solutions starting from the compact set $K \subset \mathbb{R}^{n}$ exist on $[0, T]$. Then $\mathcal{S}([0, T], K)$ is compact for the topology of uniform convergence on $[0, T]$.

\section{Homogeneity}

We shall now present the geometric homogeneity, which needs first the notion of Euler vector field to be defined.

Definition 2.4: [11] A vector field $\nu \in \mathcal{C}^{1}\left(\mathbb{R}^{n}, \mathbb{R}^{n}\right)$ is said to be Euler if it is complete and if the origin is a GAS equilibrium of $-\nu$. We will always write $\Phi$ the flow of $\nu$, that is $\Phi^{s}(x)$ is the current state at time $s$ of the trajectory of $\nu$ starting from $x$ at $s=0$.

Definition 2.5: Let $\nu$ be an Euler vector field.

- A function $V: \mathbb{R}^{n} \rightarrow \mathbb{R}$ is $\nu$-homogeneous of degree $\kappa \in \mathbb{R}$ if:

$$
V\left(\Phi^{s}(x)\right)=e^{\kappa s} V(x) \quad \forall x \in \mathbb{R}^{n}, \forall s \in \mathbb{R} .
$$

- A vector field $f: \mathbb{R}^{n} \rightarrow \mathbb{R}^{n}$ is $\nu$-homogeneous of degree $\kappa \in \mathbb{R}$ if:

$$
f\left(\Phi^{s}(x)\right)=e^{\kappa s} d_{x} \Phi^{s} f(x) \quad \forall x \in \mathbb{R}^{n}, \forall s \in \mathbb{R} .
$$

The relation (3) can be recast under the more compact form $\mathcal{H}_{\kappa}^{s}(f)=f$, where the vector field $\mathcal{H}_{\kappa}^{s}(f)$ is defined by:

$$
\mathcal{H}_{\kappa}^{s}(f): x \mapsto e^{-\kappa s}\left(d_{x} \Phi^{s}\right)^{-1} f\left(\Phi^{s}(x)\right) .
$$

Consider now a homogeneous vector field $f$ such that the following equation admits solutions:

$$
\dot{x}=f(x) .
$$

Then the homogeneity property of $f$ induce a very important property on the solutions of (5).

Proposition 2.6: [12] Assume that the vector field $f: \mathbb{R}^{n} \rightarrow$ $\mathbb{R}^{n}$ is $\nu$-homogeneous of degree $\kappa$. For any solution $x(t)$ of (5) and for all $s \in \mathbb{R}$, the curve $t \mapsto \Phi^{s}\left(x\left(e^{\kappa s} t\right)\right)$ is a solution of (5). If the system (5) admits a (semi-)flow $\Psi^{t}(x)$, we have

$$
\Phi^{s} \circ \Psi^{e^{\kappa s} t}=\Psi^{t} \circ \Phi^{s} .
$$

\section{HOMOGENEOUS DIFFERENTIAL INCLUSIONS}

In this section, we continue to consider the DI (2) for which the standard assumptions hold. Our aim is to define a homogeneity notion consistent with the conventional definition and see how the nice properties of the homogeneous continuous systems can be generalized. We adopt hereafter a natural definition, which is also a straightforward extension of the definition of [8] (given only for weighted homogeneity).

Definition 3.1: Let $\nu$ be an Euler vector field. A set-valued $\operatorname{map} F: \mathbb{R}^{n} \rightrightarrows \mathbb{R}^{n}$ is $\nu$-homogeneous of degree $\kappa \in \mathbb{R}$ if for all $s \in \mathbb{R}$ we have $\mathcal{H}_{\kappa}^{s}(F)=F$, where we extend the operator $\mathcal{H}_{\kappa}^{s}$ defined in (4) by $\mathcal{H}_{\kappa}^{s}(F): x \mapsto e^{-\kappa s}\left(d_{x} \Phi^{s}\right)^{-1} F\left(\Phi^{s}(x)\right)$.

The following lemma is a result of an easy application of the definitions.

Lemma 3.2: Let $F$ be a set-valued map satisfying the standard assumptions. Then for all $\kappa \in \mathbb{R}$ and all $s \in \mathbb{R}$ the set-valued map $\mathcal{H}_{\kappa}^{s}(F)$ satisfies the standard assumptions.

The following Proposition is an extension of Proposition 2.6.

Proposition 3.3: Let $F$ be a $\nu$-homogeneous set-valued map of degree $\kappa$, satisfying the standard assumptions. Then for all $x_{0} \in \mathbb{R}^{n}$ and any solution $x$ of the system (2) starting at $x_{0}$ 
and all $s \in \mathbb{R}$, the absolute continuous curve $t \mapsto \Phi^{s}\left(x\left(e^{\kappa s} t\right)\right)$ is a solution of the system (2) starting at $\Phi^{s}\left(x_{0}\right)$.

Proof. Consider a solution $x$ of (2) starting at $x_{0}$. The curve $t \mapsto \Phi^{s}\left(x\left(e^{\kappa s} t\right)\right)$ is clearly an absolute continuous curve for all $s \in \mathbb{R}$. Moreover, for almost all $t \in \mathbb{R}$ we have:

$$
\begin{aligned}
\frac{d}{d t} \Phi^{s}\left(x\left(e^{\kappa s} t\right)\right) & =e^{\kappa s} d_{x\left(e^{\kappa s} t\right)} \Phi^{s} \dot{x}\left(e^{\kappa s} t\right) \\
& \in e^{\kappa s} d_{x\left(e^{\kappa s} t\right)} \Phi^{s} F\left(x\left(e^{\kappa s} t\right)\right) .
\end{aligned}
$$

Since $F$ is $\nu$-homogeneous of degree $\kappa$, we find that $\frac{d}{d t} \Phi^{s}\left(x\left(e^{\kappa s} t\right)\right) \in F\left(\Phi^{s}\left(x\left(e^{\kappa s} t\right)\right)\right)$ and thus the curve $t \mapsto$ $\Phi^{s}\left(x\left(e^{\kappa s} t\right)\right)$ is a solution of (2) for all $s \in \mathbb{R}$.

Remark 3.4: This proposition may also be recast using the generalized flow, stating that, for all $t \geq 0$, for all $s \in \mathbb{R}$ and all compact sets $K \subset \mathbb{R}^{n}$ :

$$
\Psi^{t}\left(\Phi^{s}(K)\right)=\Phi^{s}\left(\Psi^{e^{\kappa s}} t(K)\right) .
$$

As we have seen, in many situations, the set-valued map $F$ comes from the Filippov's regularization procedure of a discontinuous vector field $f$. Suppose that we have a vector field $f$, which is homogeneous in the sense of Definition 2.5. If we apply the regularization procedure, is the homogeneity property preserved in the sense of Definition 3.1? The answer is positive as shown in the Corollary 3.6.

Proposition 3.5: Let $f \in \mathcal{L}_{\text {loc }}^{\infty}\left(\mathbb{R}^{n}, \mathbb{R}^{n}\right)$ be a vector field. Then $\mathcal{H}_{\kappa}^{s}(\mathcal{F}[f])=\mathcal{F}\left[\mathcal{H}_{\kappa}^{s}(f)\right]$ holds for all $(s, \kappa) \in \mathbb{R} \times \mathbb{R}$.

Proof. Since for all $\varepsilon>0$ there exist $\varepsilon_{-}>0$ and $\varepsilon_{+}>0$ such that $\Phi^{s}\left(B\left(x, \varepsilon_{-}\right)\right) \subset B\left(\Phi^{s}(x), \varepsilon\right) \subset \Phi^{s}\left(B\left(x, \varepsilon_{+}\right)\right)$we have $\mathcal{F}[f]\left(\Phi^{s}(x)\right)=$

$$
\begin{aligned}
& \bigcap_{\varepsilon>0} \bigcap_{N \in \mathcal{N}} \overline{\operatorname{conv}}\left(f(y), y \in B\left(\Phi^{s}(x), \varepsilon\right) \backslash N\right) \\
= & \bigcap_{\varepsilon>0} \bigcap_{N \in \mathcal{N}} \overline{\operatorname{conv}}\left(f(y), y \in \Phi^{s}(B(x, \varepsilon)) \backslash N\right) \\
= & \bigcap_{\varepsilon>0} \bigcap_{N \in \mathcal{N}} \overline{\operatorname{conv}}\left(f\left(\Phi^{s}(z)\right), z \in B(x, \varepsilon) \backslash N\right)
\end{aligned}
$$

Hence we find that $\mathcal{H}_{\kappa}^{s}(\mathcal{F}[f])(x)=$

$$
\bigcap_{\varepsilon>0} \bigcap_{N \in \mathcal{N}} \overline{\operatorname{conv}}\left(\left(d_{x} \Phi^{s}\right)^{-1} d_{z} \Phi^{s} \mathcal{H}_{\kappa}^{s}(f)(z), z \in B(x, \varepsilon) \backslash N\right) \text {. }
$$

Let us denote by $\sigma_{\max }\left(\left(d_{x} \Phi^{s}\right)^{-1} d_{z} \Phi^{s}\right)$ the biggest singular value of the linear mapping $\left(d_{x} \Phi^{s}\right)^{-1} d_{z} \Phi^{s}$. The function $\varphi$ : $z \mapsto\left|\sigma_{\max }\left(\left(d_{x} \Phi^{s}\right)^{-1} d_{z} \Phi^{s}\right)-1\right|$ is continuous and therefore bounded on $B(x, \varepsilon)$ and moreover vanishes at $z=x$. For all $z \in B(x, \varepsilon)$ we have:

$$
\left\|\left(d_{x} \Phi^{s}\right)^{-1} d_{z} \Phi^{s} \mathcal{H}_{\kappa}^{s}(f)(z)-\mathcal{H}_{\kappa}^{s}(f)(z)\right\| \leq M(\varepsilon),
$$

where the function $M$ is defined by $M(0)=0$ and for $\varepsilon \neq 0$ by $M(\varepsilon)=\sup _{z \in B(x, \varepsilon)} \varphi(z) \operatorname{ess} \sup _{z \in B(x, \varepsilon)}\left\|\mathcal{H}_{\kappa}^{s}(f)(z)\right\|$. The function $M$ is continuous at zero. We have proved that $\left(d_{x} \Phi^{s}\right)^{-1} d_{z} \Phi^{s} \mathcal{H}_{\kappa}^{s}(f)(z) \in \mathcal{H}_{\kappa}^{s}(f)(z)+B(0, M(\varepsilon))$. It follows that $\mathcal{H}_{\kappa}^{s}(\mathcal{F}[f])(x) \subset$

$$
\begin{aligned}
& \bigcap_{\varepsilon>0} \bigcap_{N \in \mathcal{N}} \overline{\operatorname{conv}}\left(\mathcal{H}_{\kappa}^{s}(f)(z)+B(0, M(\varepsilon)),\right. \\
& z \in B(x, \varepsilon) \backslash N) \\
= & \bigcap_{\varepsilon>0}\left[\left(\bigcap_{N \in \mathcal{N}} \overline{\operatorname{conv}}\left(\mathcal{H}_{\kappa}^{s}(f)(z), z \in B(x, \varepsilon) \backslash N\right)\right)\right. \\
& +B(0, M(\varepsilon))] \\
= & \bigcap_{\varepsilon>0} \bigcap_{N \in \mathcal{N}} \overline{\operatorname{conv}}\left(\mathcal{H}_{\kappa}^{s}(f)(z), z \in B(x, \varepsilon) \backslash N\right) \\
= & \mathcal{F}\left[\mathcal{H}_{\kappa}^{s}(f)\right](x) .
\end{aligned}
$$

The proof of the converse inclusion is similar.

Corollary 3.6: Let $f \in \mathcal{L}_{\text {loc }}^{\infty}\left(\mathbb{R}^{n}, \mathbb{R}^{n}\right)$ be a vector field. Suppose $f$ is $\nu$-homogeneous of degree $\kappa$. Then $\mathcal{F}(f)$ is $\nu$ homogeneous of degree $\kappa$.
Proof. Since $f$ is $\nu$-homogeneous of degree $\kappa$, we have $\mathcal{H}_{\kappa}^{s}(f)=f$. Hence $\mathcal{F}(f)=\mathcal{F}\left(\mathcal{H}_{\kappa}^{s}(f)\right)=\mathcal{H}_{\kappa}^{s}(\mathcal{F}(f))$ by Proposition 3.5 and therefore $\mathcal{F}(f)$ is $\nu$-homogeneous of degree $\kappa$.

Example 3.7: Consider the $n$-integrator with an input given by $u(x)=-\sum_{i} k_{i} \operatorname{sign}\left(x_{i}\right), k_{i}>0$ :

$$
\left\{\begin{array}{lll}
\dot{x}_{1} & = & x_{2} \\
\vdots & & \vdots \\
\dot{x}_{n-1} & = & x_{n} \\
\dot{x}_{n} & = & -\sum_{i} k_{i} \operatorname{sign}\left(x_{i}\right)
\end{array} .\right.
$$

It is easy to check that this vector field is $\nu$-homogeneous of degree -1 with $\nu(x)=\left(n x_{1}, \ldots, 2 x_{n-1}, x_{n}\right)^{T}$. The associated differential inclusion is therefore $\nu$-homogeneous of degree -1 as well.

\section{QUALITATIVE RESULTS ON HOMOGENEOUS DISCONTINUOUS SYSTEMS}

In the previous section, we have seen how to define a homogeneous discontinuous system and the basic properties stemming from this definition. But the classical theory of homogeneity highlights a lot of very important and useful properties of homogeneous systems. Among those:

- the Theorem of Rosier [13], which is a homogeneous converse Lyapunov theorem;

- the link between negative degree of homogeneity and finite-time stability [14], and the properties of the settlingtime function [15];

- the consequences of the existence of a strictly positively invariant compact set [16];

- the equivalence of the notions of local attractivity and global stability for homogeneous systems [6], [16].

We shall generalize these properties in this section.

\section{A. Converse Lyapunov theorem for homogeneous differential inclusions}

The following theorem asserts that a strongly globally asymptotically stable (i.e. asymptotic stability property holds for all solutions initiated from all initial conditions) homogeneous differential inclusion admits a homogeneous Lyapunov function. This result is a generalization of the theorem independently proved by Rosier [13] and Zubov [17] for continuous systems and of its extension, given in [18], where weighted homogeneous locally essentially bounded vector fields were considered and the existence of a merely continuous Lyapunov function was proved.

Theorem 4.1: Let $F$ be a $\nu$-homogeneous set-valued map of degree $\kappa$, satisfying the standard assumptions. Then the following statements are equivalent:

- The origin is (strongly) GAS for the system (2).

- For all $\mu>\max (-\kappa, 0)$, there exists a pair $(V, W)$ of continuous functions, such that:

1) $V$ is of class $\mathcal{C}^{\infty}\left(\mathbb{R}^{n}, \mathbb{R}\right), V$ is positive definite and $\nu$-homogeneous of degree $\mu$;

2) $W$ is of class $\mathcal{C}^{\infty}\left(\mathbb{R}^{n} \backslash\{0\}, \mathbb{R}\right), W$ is strictly positive outside the origin and $\nu$-homogeneous of degree $\mu+$ $\kappa ;$ 
3) $\max _{v \in F(x)} d_{x} V v \leq-W(x)$ for all $x \neq 0$.

Proof. By the result of [2], the two following statements are equivalent:

- The system (2) is strongly GAS.

- There exist a pair $\left(V_{0}, W_{0}\right)$ of continuous functions, such that:

1) $V_{0} \in \mathcal{C}^{\infty}\left(\mathbb{R}^{n}\right), V_{0}$ is positive definite;

2) $W_{0} \in \mathcal{C}^{\infty}\left(\mathbb{R}^{n} \backslash\{0\}\right), W_{0}$ is strictly positive outside the origin;

3) $\max _{v \in F(x)} d_{x} V_{0} v \leq-W_{0}(x)$ for all $x \neq 0$.

Hence, it suffices to prove that the homogeneity condition allows us to build a homogeneous Lyapunov pair. The sequel of the proof is widely inspired by the proof from [13]. Let $a:\left[0,+\infty\left[\rightarrow[0,1]\right.\right.$ be a $\mathcal{C}^{\infty}$ function such that for all $t \leq 1$, $a(t)=0$, for all $t \geq 2, a(t)=1$ and for all $t \in] 1,2\left[, a^{\prime}(t)>0\right.$. Set $\mu>\max (-\kappa, 0)$ and:

$$
V(x)=\int_{\mathbb{R}} e^{-\mu s} a\left(V_{0}\left(\Phi^{s}(x)\right)\right) d s,
$$

then $V(0)=0$. For all $x \neq 0$, there exists $s_{1}$ such that for all $s \leq s_{1}, V_{0}\left(\Phi^{s}(x)\right) \leq 1$. Similarly, there exists $s_{2}$ such that for all $s \geq s_{2}, V_{0}\left(\Phi^{s}(x)\right) \geq 2$. Hence:

$$
V(x)=\int_{s_{1}}^{s_{2}} e^{-\mu s} a\left(V_{0}\left(\Phi^{s}(x)\right)\right) d s+\frac{e^{-\mu s_{2}}}{\mu},
$$

and $V$ is well-defined.

The homogeneity of $V$ is straightforward using a change of variable: $V\left(\Phi^{\sigma}(x)\right)=\int_{\mathbb{R}} e^{-\mu s} a\left(V_{0}\left(\Phi^{s}\left(\Phi^{\sigma}(x)\right)\right)\right) d s=$ $e^{\mu \sigma} \int_{\mathbb{R}} e^{-\mu u} a\left(V_{0}\left(\Phi^{u}(x)\right)\right) d u=e^{\mu \sigma} V(x)$.

On the other hand, for all $s \in \mathbb{R}, e^{-\mu s} a\left(V_{0}\left(\Phi^{s}(x)\right)\right)$ is $\mathcal{C}^{\infty}$ and $\left|e^{-\mu s} a\left(V_{0}\left(\Phi^{s}(x)\right)\right)\right| \leq e^{-\mu s}$ which is integrable $(\mu>0)$. Thus $V$ belongs to the class $\mathcal{C}^{\infty}$ on $\mathbb{R}^{n}$ and therefore proper [16]. Moreover, for all $v \in F(x)$ :

$$
d_{x} V v=\int_{\mathbb{R}} e^{-\mu s} a^{\prime}\left(V_{0}\left(\Phi^{s}(x)\right)\right)\left(d_{\Phi^{s}(x)} V_{0}\right)\left(d_{x} \Phi^{s}\right) v d s .
$$

As $F$ is homogeneous, there exists $\tilde{v} \in F\left(\Phi^{s}(x)\right)$ such that $\tilde{v}=e^{\kappa s} d_{x} \Phi^{s} v$. Hence:

$$
\begin{aligned}
d_{x} V v & =\int_{\mathbb{R}} e^{-(\mu+\kappa) s} a^{\prime}\left(V_{0}\left(\Phi^{s}(x)\right)\right)\left(d_{\Phi^{s}(x)} V_{0}\right) \tilde{v} d s \\
& \leq-\int_{\mathbb{R}} e^{-(\mu+\kappa) s} a^{\prime}\left(V_{0}\left(\Phi^{s}(x)\right)\right) W_{0}\left(\Phi^{s}(x)\right) d s .
\end{aligned}
$$

Let us denote:

$$
W(x)=\int_{\mathbb{R}} e^{-(\mu+\kappa) s} a^{\prime}\left(V_{0}\left(\Phi^{s}(x)\right)\right) W_{0}\left(\Phi^{s}(x)\right) d s,
$$

thus $\max _{v \in F(x)} d_{x} V v \leq-W(x)$. It is clear that $W$ is well-defined and strictly positive. The function $W$ is clearly homogeneous of degree $\kappa+\mu$ ( this fact can be also proven using a simple change of variable). Moreover, for all $s \in \mathbb{R}$, the function $x \mapsto e^{-(\mu+\kappa) s} a^{\prime}\left(V_{0}\left(\Phi^{s}(x)\right)\right) W_{0}\left(\Phi^{s}(x)\right)$ is of class $\mathcal{C}^{\infty}\left(\mathbb{R}^{n} \backslash\{0\}\right)$. Let us show that

$$
\xi(x, s)=\left|e^{-(\mu+\kappa) s} a^{\prime}\left(V_{0}\left(\Phi^{s}(x)\right)\right) W_{0}\left(\Phi^{s}(x)\right)\right|
$$

is locally upper-bounded by an integrable function. Set $U_{x}=$ $\bar{B}(x,|x| / 2)$. For $x \neq 0, U_{x}$ is a neighborhood of $x$. Since $\nu$ is Euler, there exists $s_{1}, s_{2}$ such that for all $y \in U_{x} \subset \mathbb{R}^{n} \backslash\{0\}$, for all $s \leq s_{1}, V_{0}\left(\Phi^{s}(y)\right) \leq 1$ and for all $s \geq s_{2}, V_{0}\left(\Phi^{s}(y)\right) \geq$ 2. Hence $a^{\prime}\left(V_{0}\left(\Phi^{s}(y)\right)\right)=0$ for all $\left.s \notin\right] s_{1}, s_{2}$ [ and for all $y \in$ $U_{x}$. Denote $c_{1}=\sup _{y \in U_{x}} \sup _{s \in\left[s_{1}, s_{2}\right]} W_{0}\left(\Phi^{s}(y)\right)$ and $c_{2}=$ $\sup _{t \in \mathbb{R}} a^{\prime}(t)$. We get $\xi(x, s) \leq e^{-(\mu+\kappa) s} \mathbf{1}_{U_{x}}(x) c_{1} c_{2}$ which is clearly integrable (with $\mathbf{1}_{A}(x)=1$ if $x \in A$ and 0 else). Therefore, $W$ is $\mathcal{C}^{\infty}$ on a neighborhood of $x$ for all $x \in$ $\mathbb{R}^{n} \backslash\{0\}$, i.e. $W$ is $\mathcal{C}^{\infty}$ on $\mathbb{R}^{n} \backslash\{0\}$.

The only point remaining to prove is the continuity of $W$ at the origin. Let $\varepsilon>0$ be fixed. There exists $s_{1}$ such that for all $s \leq s_{1}, V_{0}\left(\Phi^{s}(y)\right) \leq 1$ for all $y \in B(0, \varepsilon)$. Thus, introducing the sets $\mathcal{A}=\left\{V_{0}\left(\Phi^{s}(y)\right)>2\right\}$ and $\mathcal{B}=\left\{V_{0}\left(\Phi^{s}(y)\right) \leq 2\right\}$, for all $y \in B(0, \varepsilon)$, we have

$$
\begin{aligned}
W(y) & =\int_{s_{1}}^{+\infty} e^{-(\mu+\kappa) s} a^{\prime}\left(V_{0}\left(\Phi^{s}(y)\right)\right) W_{0}\left(\Phi^{s}(y)\right) d s \\
& =\int_{s_{1}}^{+\infty} e^{-(\mu+\kappa) s} a^{\prime}\left(V_{0}\left(\Phi^{s}(y)\right)\right) W_{0}\left(\Phi^{s}(y)\right) \mathbf{1}_{\mathcal{A}} d s \\
& +\int_{s_{1}}^{+\infty} e^{-(\mu+\kappa) s} a^{\prime}\left(V_{0}\left(\Phi^{s}(y)\right)\right) W_{0}\left(\Phi^{s}(y)\right) \mathbf{1}_{\mathcal{B}} d s .
\end{aligned}
$$

Since $a^{\prime}(t)=0$ for $t>2$ the first part vanishes. But $V_{0}$ is proper, thus $\mathcal{B}$ is compact and $W_{0}$ is bounded by $c_{3}>0$ on this set. Therefore

$$
\begin{aligned}
W(y) & =\int_{s_{1}}^{+\infty} e^{-(\mu+\kappa) s} a^{\prime}\left(V_{0}\left(\Phi^{s}(y)\right)\right) W_{0}\left(\Phi^{s}(y)\right) \mathbf{1}_{\mathcal{B}} d s \\
& \leq \int_{s_{1}}^{+\infty} e^{-(\mu+\kappa) s} c_{2} c_{3} d s<+\infty,
\end{aligned}
$$

since $\mu+\kappa>0$. Finally, since $W$ is homogeneous, it is also continuous at the origin and the proof is completed.

\section{B. Application to Finite-Time Stability}

In this subsection, we aim at applying the Theorem 4.1 to Finite-Time Stability (FTS). Indeed, the existence of a smooth homogeneous Lyapunov function provides informations about the rate of convergence of such systems.

Definition 4.2: Consider the system (2). The origin is said to be FTS if:

1) the origin is (strongly) stable;

2) there exists an open neighborhood $\mathcal{U}$ of the origin such that for all $x \in \mathcal{U}$, there exists $\tau \geq 0$ such that for all $t \geq \tau$, we have $\Psi^{t}(x)=\{0\}$ (strong finite-time convergence).

The settling-time function is then defined for $x \in \mathcal{U}$ by $\mathrm{T}(x)=\inf \left\{\tau \geq 0: \forall t \geq \tau, \Psi^{t}(x)=\{0\}\right\}$.

If the neighborhood $\mathcal{U}$ can be chosen to be $\mathbb{R}^{n}$, the system is said to be Globally FTS (GFTS).

Corollary 4.3: Let $F$ be a $\nu$-homogeneous set-valued map of degree $\kappa<0$, satisfying the standard assumptions. Assume also that the origin is GAS for $F$. Then the origin is GFTS for $F$ and the settling-time function is continuous at zero and locally bounded.

Proof. The origin is GAS for $F$ and $F$ is homogeneous; thus by Theorem 4.1, $F$ admits a homogeneous Lyapunov pair $(V, W)$. Let us apply Lemma 4.2 of [16] to the continuous functions $V$ and $W$. We get that for all $x \in \mathbb{R}^{n} \backslash\{0\}$, and for all $v \in F(x)$ :

$$
d_{x} V v \leq-W(x) \leq-C(V(x))^{\frac{\kappa+\mu}{\mu}},
$$

where $C=\min _{\{V=1\}} W$. Since $\frac{\kappa+\mu}{\mu}<1, V$ converges to zero in a finite time, giving us the finite-time convergence of the system, which is therefore GFTS. Moreover, a direct 
integration of the inequation (8) gives $\mathrm{T}(x) \leq \frac{\mu V(x)^{\frac{-\kappa}{\mu}}}{-\kappa C}$, where $\mathrm{T}$ denotes the settling-time function. Since $V$ is continuous, $\mathrm{T}$ is locally bounded and continuous at zero.

It has been shown in [16] that under the assumptions of homogeneity (of negative degree), continuity of the right-hand side and forward uniqueness of solutions, the settling-time function of a finite-time stable system is continuous. The two latter do obviously not hold in our context. We have seen that, however, under the standard assumptions, the settlingtime function remains continuous at the origin and locally bounded. Let us emphasize that these conclusions are sharp and that the settling-time function is not continuous in general. See, for instance, [19] or the following example.

Example 4.4: (A counterexample to the second statement of Theorem 1 from [8]) Consider the system defined on $\mathbb{R}^{2}$ by:

$$
\dot{x}=-\left(\operatorname{sign}\left(x_{1}\right)+2\right) \frac{x}{\|x\|} \text {. }
$$

This system is clearly strongly (uniformly [8]) GFTS and $\nu$ homogeneous of negative degree with $\nu=x_{1} \frac{\partial}{\partial x_{1}}+x_{2} \frac{\partial}{\partial x_{2}}$. A simple computation shows that the settling-time function is:

$$
\mathrm{T}(x)=\left\{\begin{array}{ll}
\|x\| & x_{1} \geq 0 \\
\frac{\|x\|}{3} & x_{1}<0
\end{array},\right.
$$

which is discontinuous on $x_{1}=0$.

\section{Sufficient conditions for Global Asymptotic Stability}

In this subsection, we focus on the qualitative properties of homogeneous discountinuous systems that can lead to GAS. The first result is a generalization of Theorem 6.1 of [16].

Theorem 4.5: Suppose that $K$ is a strongly strictly positively invariant compact subset (SPI) of $\mathbb{R}^{n}$ for the homogeneous system (2). Then the origin is GAS for (2).

Proof. Let us denote $\kappa$ the degree of $F$. Since the solutions starting in $K$ are bounded, they are defined for all $t \geq 0$, and thus $\Psi^{t}(K)$ is compact for all $t>0$ by Theorem 2.2. From equation (7), we have ( $\stackrel{\circ}{K}$ defines interior for a set $K \subset \mathbb{R}^{n}$ ):

$$
\Psi^{t}\left(\Phi^{s}(K)\right)=\Phi^{s}\left(\Psi^{e^{\kappa s} t}(K)\right) \subset \Phi^{s}(\stackrel{\circ}{K})=\overbrace{\Phi^{s}(K)}^{\overbrace{}^{\circ}} .
$$

Therefore, the set $\Phi^{s}(K)$ is SPI for all $s \in \mathbb{R}$. We also note that $\Psi^{s+t}(K)=\Psi^{t}\left(\Psi^{s}(K)\right) \subset \Psi^{t}(K)$. Thus $\left(\Psi^{t}(K)\right)_{t>0}$ is a nested family of compact sets and $K_{\infty}=\cap_{t \geq 0} \Psi^{t}(K)$ is a non-empty compact; it is also the biggest positively invariant compact subset of $K$. But for all $s \in \mathbb{R}$

$$
\Phi^{s}\left(K_{\infty}\right)=\bigcap_{t \geq 0} \Phi^{s}\left(\Psi^{t}(K)\right)=\bigcap_{\tau \geq 0} \Psi^{\tau}\left(\Phi^{s}(K)\right)
$$

has the same property. Therefore $K_{\infty}=\Phi^{s}\left(K_{\infty}\right)$, that is $K_{\infty}$ is an invariant subset for $\Phi$. Since $\nu$ is Euler, we conclude that $K_{\infty}=\{0\}$ and every solution starting in $K$ converges to the origin, thus $0 \in K$. The stability follows from the SPI of the sets $\Phi^{s}(K)$ for all $s \in \mathbb{R}$.

Let us illustrate how this Theorem can be used to derive robustness properties for some homogeneous systems.

Example 4.6: It is known that for $k_{1}>k_{2}>0$, the following system is GAS:

$$
\left\{\begin{array}{l}
\dot{x}_{1}=x_{2} \\
\dot{x}_{2}=-k_{1} \operatorname{sign}\left(x_{1}\right)-k_{2} \operatorname{sign}\left(x_{2}\right)
\end{array} .\right.
$$

As we have seen, this system is $\nu$-homogeneous of degree -1 with $\nu(x)=\left(2 x_{1}, x_{2}\right)^{T}$. Therefore, there exists a $\nu$ homogeneous Lyapunov pair $(V, W)$ of degrees $\kappa>1$ and $\kappa-1$. We denote $F_{0}$ the set valued map associated to this vector field and for $\alpha \in \mathbb{R}^{2}$ we denote $F_{\alpha}$ the set valued map associated to the vector field:

$$
\left\{\begin{array}{l}
\dot{x}_{1}=x_{2} \\
\dot{x}_{2}=-\left(k_{1}+\alpha_{1}\right) \operatorname{sign}\left(x_{1}\right)-\left(k_{2}+\alpha_{2}\right) \operatorname{sign}\left(x_{2}\right)
\end{array}\right.
$$

We shall prove that the compact set $K=\left\{x \in \mathbb{R}^{2}: V(x) \leq\right.$ $1\}$ is SPI for $F_{\alpha}$, for small values of $\alpha$. Let $y \in K$ and $v \in F_{\alpha}(y)$. There exists $x \in S=\left\{x \in \mathbb{R}^{n}: V(x)=1\right\}$ and $s \in \mathbb{R}$ such that $\Phi^{s}(x)=y$. By homogeneity, there also exists $w \in F_{\alpha}(x)$ such that $v=e^{-s} d_{x} \Phi^{s} w$. Therefore:

$$
d_{y} V v=d_{\Phi^{s}(x)} V e^{-s} d_{x} \Phi^{s} w=e^{(\kappa-1) s} d_{x} V w .
$$

Since $w \in F_{\alpha}(x)$, there exist $\sigma_{1}, \sigma_{2} \in[-1,1]$ such that $w=\left(x_{2},-\left(k_{1}+\alpha_{1}\right) \sigma_{1}-\left(k_{2}+\alpha_{2}\right) \sigma_{2}\right)^{T}$. Let us denote $\tilde{w}=\left(x_{2},-k_{1} \sigma_{1}-k_{2} \sigma_{2}\right)^{T} \in F_{0}(x)$. We have:

$$
\begin{aligned}
d_{y} V v & =e^{(\kappa-1) s} d_{x} V w \\
& =e^{(\kappa-1) s}\left[d_{x} V \tilde{w}+d_{x} V(w-\tilde{w})\right] \\
& \leq e^{(\kappa-1) s}\left[-W(x)+\sup _{x \in S}\left\|d_{x} V\right\| \cdot\|w-\tilde{w}\| \|\right] \\
& \leq e^{(\kappa-1) s}\left[-\inf _{x \in S} W(x)+\sup _{x \in S}\left\|d_{x} V\right\| \cdot\left\|\sum_{i=1}^{2} \alpha_{i} \sigma_{i}\right\|\right] \\
& \leq e^{(\kappa-1) s}\left[-\inf _{x \in S} W(x)+\sup _{x \in S}\left\|d_{x} V\right\| \cdot \sum_{i=1}^{2}\left\|\alpha_{i}\right\|\right] .
\end{aligned}
$$

Therefore, if $\left|\alpha_{1}\right|+\left|\alpha_{2}\right|<\frac{\inf _{x \in S} W(x)}{\sup _{x \in S}\left\|d_{x} V\right\|}, d_{y} V v<0$, which means that $K$ is strictly positively invariant. The set valued map $F_{\alpha}$ being homogeneous, it is hence GAS for $\alpha$ small enough. Finally, any stabilizing control under the form $u(x)=$ $-k_{1} \operatorname{sign}\left(x_{1}\right)-k_{2} \operatorname{sign}\left(x_{2}\right)$ is robust w.r.t. small time-varying errors on the gains $k_{i}$, like implementation errors.

As we have seen in the introduction, for continuous homogeneous systems (with forward uniqueness of solutions) the notions of local attractiveness and global asymptotic stability are merged. This fact admits a generalization in the discontinuous setting.

Theorem 4.7: Let $F$ be a $\nu$-homogeneous set-valued map of degree $\kappa$ with the standard assumptions. Assume moreover that all the solutions of the associated DI are defined for all $t \geq 0$ and tend to 0 when $t \rightarrow \infty$. Then the origin is strongly globally asymptotically stable.

Proof. By contradiction, assume that the origin is unstable. Then there exists a neighborhood $\mathcal{U}$ of the origin such that for all neighborhoods of the origin $\mathcal{V} \subset \mathcal{U}$, there exists a solution starting in $\mathcal{V}$ which does not stay in $\mathcal{U}$. Taking $\mathcal{V}=B(0,1 / i)$, there exists a solution $x_{i}$ such that $x_{i}(0) \in \mathcal{V}$, and there exists a real number $t_{i}$ such that $x_{i}\left(t_{i}\right) \notin \mathcal{U}$. Therefore, when $i \rightarrow \infty$, $x_{i}(0) \rightarrow 0$ but $\left(x_{i}\left(t_{i}\right)\right)$ does not converge to 0 .

Let us denote by $N$ a $\nu$-homogeneous norm and denote $\delta_{i}=N\left(x_{i}(0)\right)$. There exists $\varepsilon>0$ such that $N\left(x_{i}\left(t_{i}\right)\right) \geq \varepsilon$. We can also assume that $\delta_{i}<\varepsilon$. Since $x_{i}\left(t_{i}\right)>0$, we can finally assume that $\delta_{i}>0$ by continuity. 
Let us denote:

$$
\begin{aligned}
a_{i} & =\sup \left\{t \in\left[0, t_{i}\right]: N\left(x_{i}(t)\right)=\delta_{i}\right\}, \\
b_{i} & =\inf \left\{t \in\left[0, t_{i}\right]: N\left(x_{i}(t)\right)=\varepsilon\right\} .
\end{aligned}
$$

We define $y_{i}(t)=x_{i}\left(t+a_{i}\right)$. The curves $y_{i}$ are solutions of (2) defined on $\left[0, b_{i}-a_{i}\right]$ and we have $N\left(y_{i}(0)\right)=\delta_{i}, N\left(y_{i}(t)\right) \in$ $\left(\delta_{i}, \varepsilon\right)$ for all $t \in\left(0, b_{i}-a_{i}\right)$ and $N\left(y_{i}\left(b_{i}-a_{i}\right)\right)=\varepsilon$.

By Proposition 3.3, for all $s \in \mathbb{R}$, the curve $t \mapsto$ $\Phi^{s}\left(y_{i}\left(e^{\kappa s} t\right)\right)$ is a solution. Set $s_{i}=-\ln \delta_{i}, z_{i}(t)=$ $\Phi^{s_{i}}\left(y_{i}\left(e^{\kappa s_{i}} t\right)\right)$ and $t_{i}^{*}=\delta_{i}^{\kappa}\left(b_{i}-a_{i}\right)$. We find $N\left(z_{i}(t)\right)=$ $e^{s_{i}} N\left(y_{i}\left(e^{\kappa s_{i}} t\right)\right)=N\left(y_{i}\left(e^{\kappa_{i}} t\right)\right) / \delta_{i}$. Hence $N\left(z_{i}(0)\right)=1$, for all $t \in\left(0, t_{i}^{*}\right)$ we have $N\left(z_{i}(t)\right) \geq 1$ and $N\left(z_{i}\left(t_{i}^{*}\right)\right)=\varepsilon / \delta_{i}$.

Assume that there exists a bounded subsequence of $\left(t_{i}^{*}\right)$. By Theorem 2.2, the corresponding subsequence $\left(z_{j}\left(t_{j}^{*}\right)\right)$ is bounded; however $N\left(z_{j}\left(t_{j}^{*}\right)\right)=\frac{\varepsilon}{\delta_{j}} \rightarrow \infty$. Then the sequence $\left(t_{i}\right)$ tends to $+\infty$.

By Corollary 2.3, let us now extract a subsequence $\left(z_{\varphi_{1}(i)}\right)$ converging to a solution $\bar{z}$ on $[0,1]$. Then we extract a subsubsequence $\left(z_{\varphi_{1} \circ \varphi_{2}(i)}\right)$ converging to $\bar{z}$ on $[0,2]$, etc. A diagonal extraction provides us the subsequence $\left(z_{\varphi_{1} \circ \ldots \circ \varphi_{i}(i)}\right)$ which is converging to $\bar{z}$ uniformly on $[0, j]$ for all $j \in \mathbb{N}$. For all $t>0$, we have $N(\bar{z}(t))=\lim _{i} N\left(z_{\varphi_{1} \circ \ldots \circ \varphi_{i}(i)(t)}\right)$. But for $i$ large enough, we have $t \leq t_{\varphi_{1} 0 \ldots \circ \varphi_{i}(i)}^{*}$ and thus $N\left(z_{\varphi_{1} \circ \ldots \circ \varphi_{i}(i)(t)}\right) \geq 1$. Therefore $N(\bar{z}(t)) \geq 1$ and $\bar{z}(t)$ does not tend to zero, which is a contradiction.

Example 4.8: Consider a double integrator endowed with an observer written with the error equation under the form:

$$
\left\{\begin{array}{l}
\dot{x}_{1}=x_{2} \\
\dot{x}_{2}=-k_{1} \operatorname{sign}\left(x_{1}\right)-k_{2} \operatorname{sign}\left(x_{2}-e_{2}\right) \\
\dot{e}_{1}=e_{2}-l_{1}\left\lfloor e_{1} 1^{1 / 2}\right. \\
\dot{e}_{2}=-l_{2} \operatorname{sign}\left(e_{1}\right)
\end{array}\right.
$$

with $k_{1}>k_{2}>0, l_{1}, l_{2}>0$ and $\left\lfloor e_{1}\right\rceil^{1 / 2}=$ $\left|e_{1}\right|^{1 / 2} \operatorname{sign}\left(e_{1}\right)$. This system is $\nu$-homogeneous of degree -1 with $\nu\left(x_{1}, x_{2}, e_{1}, e_{2}\right)=\left(2 x_{1}, x_{2}, 2 e_{1}, e_{2}\right)^{T}$.

Consider first the error subsystem:

$$
\left\{\begin{array}{l}
\dot{e}_{1}=e_{2}-l_{1}\left\lfloor e_{1}\right\rceil^{1 / 2} \\
\dot{e}_{2}=-l_{2} \operatorname{sign}\left(e_{1}\right)
\end{array} .\right.
$$

Using the function $V(e)=l_{2}\left|e_{1}\right|+e_{2}^{2} / 2$, we see that the system (10) is FTS. Indeed, for $e_{1} \neq 0, \dot{V}(e)=-l_{1} l_{2}\left|e_{1}\right|^{1 / 2}<0$. Hence the compact set $K=\{V \leq 1\}$ is SPI, since the line $e_{1}=0$ is not invariant. Being of negative degree, we find that the system (10) is FTS.

The system (9) being clearly forward complete, it becomes equivalent in a finite time to the system:

$$
\left\{\begin{array}{l}
\dot{x}_{1}=x_{2} \\
\dot{x}_{2}=-k_{1} \operatorname{sign}\left(x_{1}\right)-k_{2} \operatorname{sign}\left(x_{2}\right)
\end{array}\right.
$$

Using the Lyapunov function $V(x)=k_{1}\left|x_{1}\right|+x_{2}^{2}$ and $k_{1}>k_{2}$, we find that the system (11) is FTS as well.

Finally, all the solutions of the system (9) converge in finitetime to 0 , but stability is not straightforward. However, the Theorem 4.7 ensures us that the stability is a consequence of the attractiveness for homogeneous systems and we find that the system (9) is GFTS.

\section{CONClusion}

In this paper, we have proposed a geometric definition of homogeneity for DI, consistent with the Filippov's regularization procedure. With this framework, we have been able to state extensions to the DI setting of results holding for continuous homogeneous systems: (i) A converse homogeneous Lyapunov theorem - if the origin is a globally asymptotically stable equilibrium, then there exists a homogeneous Lyapunov function (indeed, a homogeneous Lyapunov pair) for the DI. (ii) If the origin is a globally asymptotically stable equilibrium for a homogeneous DI of negative degree, then it is strongly FTS. (iii) The existence of a SPI compact set is equivalent to global asymptotic stability. (iv) The local attractiveness of the origin implies its global asymptotic stability.

Future works will include homogenization of a DI and study of the robustness of systems defined by homogeneous DI.

\section{REFERENCES}

[1] J. Kurzweil, "On the inversion of liapunov's second theorem on stability of motion," Translations of American Mathematical Society, vol. 24, pp. 19-77, 1963.

[2] F. H. Clarke, Y. S. Ledyaev, and R. J. Stern, "Asymptotic stability and smooth lyapunov functions," Journal of differential equations, vol. 149, pp. 69-114, 1998.

[3] M. Kawski, "Homogeneous stabilizing feedback laws," Control Theory and Advanced Technology, vol. 6, no. 4, pp. 497-516, 1990.

[4] H. Hermes, "Nilpotent approximations of control systems and distributions," SIAM Journal on Control and Optimization, vol. 24, no. 4, p. 731, 1986.

[5] S. P. Bhat and D. S. Bernstein, "Finite-time stability of continuous autonomous systems," SIAM J. Control Optim., vol. 38, no. 3, pp. 751766, 2000.

[6] A. Filippov, Differential Equations with Discontinuous Righthand Sides. Kluwer Academic, 1988.

[7] J.-P. Aubin and A. Cellina, Differential Inclusions. Grundlehren der Math. Wiisenschaften 264, Springer-Verlag, 1984.

[8] A. Levant, "Homogeneity approach to high-order sliding mode design," Automatica, vol. 41, no. 5, pp. 823-830, 2005.

[9] Y. Orlov, "Finite time stability and robust control synthesis of uncertain switched systems," SIAM J. Control Optim., vol. 43, no. 4, pp. 12531271, 2005.

[10] E. Bernuau, A. Polyakov, D. Efimov, and W. Perruquetti, "On extension of homogeneity notion for differential inclusions," in Proc. of ECC 2013, 2013.

[11] M. Kawski, "Families of dilations and asymptotic stability," in Analysis of controlled dynamical systems (Lyon, 1990), vol. 8 of Progr. Systems Control Theory, pp. 285-294, Boston, MA: Birkhäuser Boston, 1991.

[12] M. Kawski, "Geometric homogeneity and stabilization," in Proc. IFAC Nonlinear Control Symposium (A. Krener and D. Mayne, eds.), (Lake Tahoe, CA), pp. 164-169, 1995.

[13] L. Rosier, "Homogeneous Lyapunov function for homogeneous continuous vector field," Systems \& Control Letters, vol. 19, pp. 467-473, 1992.

[14] S. P. Bhat and D. S. Bernstein, "Continuous finite-time stabilization of the translational and rotational double integrator," IEEE Trans. Automat. Control, vol. 43, pp. 678-682, may 1998.

[15] S. P. Bhat and D. S. Bernstein, "Finite-time stability of homogeneous systems," in Proceedings of the American Control Conference, (Albuquerque, USA), pp. 2513-2514, 1997.

[16] S. P. Bhat and D. S. Bernstein, "Geometric homogeneity with applications to finite-time stability," Mathematics of Control, Signals and Systems, vol. 17, pp. 101-127, 2005.

[17] V. Zubov, "On ordinary differential equations with generalized homogeneous right-hand sides," Izvestiya vuzov, Matematika, vol. 1, no. 2, pp. 80-88, 1958 .

[18] L. Rosier, "Inverse of lyapunov's second theorem for measurable functions," in Proc. NOLCOS 92, (Bordeaux, France), pp. 655-660, 1992.

[19] A. Polyakov, "On settling time function and stability of vector relay systems," in International Workshop on Variable Structure Systems, pp. 149-154, IEEE, 2012. 